\title{
Chaotic Behaviour in Dynamical Systems
}

\author{
A. Hacinliyan \\ Istanbul Technical University, Istanbul, Turkey
}

Traditional undergraduate programmes in physics rely on a number of simplifications. One is the emphasis on linear systems; another is the belief that systems with a few degrees of freedom exhibit a deterministic behaviour, while those with many degrees of freedom can be treated using statistical methods only. Linear systems are attractive because they are conceptually simple and mathematically easy to solve so it is tempting to employ linear models to describe phenomena. These systems possess the important property of superposition whereby any linear combination of solutions is also a solution. Using this property one can easily fit the solution to appropriate initial conditions.

However, the simplicity of linear models seriously limits their predictive power. Since any multiple of a solution to a linear differential equation is also a solution, it is impossible to set a scale to a system described by a linear equation. Initial conditions set the scale. For instance, the differential equation describing molecular oscillations (with an amplitude of the order of Angstrom units) is the same as that for the mechanical vibrations of a macroscopic mass-spring system (with an amplitude of the order of centimeters). A nonlinear equation on the other hand, sets a scale to the physical process that it describes. For example, unitarity is a nonlinear relation involving the scattering amplitude. Indeed, there is an upper bound on scattering cross sections called the Froissart bound stipulating that total cross sections cannot increase with energy faster than the square of the logarithm. Unitarity is a key input to this upper bound.

Almost all of the interesting problems in physics involve nonlinear equations of motion. Being well-trained in the solution of linear problems, one usually tries to solve a nonlinear problem by considering it as a perturbation of an exactly solvable (and usually linear) pro-

Professor Avedis Hacinliyan is with the Department of Physics, Faculty of Science and Letters, Istanbul Technical University, MaslakIstanbul 80626 . He is mainly interested in the theoretical aspects of chaotic phenomena observed in a broad range of disciplines. blem. The convergence properties of these perturbative solutions are often not discussed. For instance, most quantum mechanics textbooks cite the anharmonic oscillator problem as an example of perturbation theory, even though it is well known that the perturbative solution based on an harmonic oscillator is divergent for all values of the perturbing quartic potential.

The two body problem (the motion of two bodies under the influence of their mutual gravitational attraction) can be solved exactly. The next simplest problem in celestial mechanics, namely the three body problem, cannot be solved exactly. A perturbative treatment based upon an expansion about a stable equilibrium point must also be used. The relation between a system of coupled nonlinear oscillators and the three body problem comes from the fact that close to points of stable equilibrium, dynamical systems can be approximated as oscillators. However, nonlinear oscillators involve frequency addition and the shifting of frequencies as a function of amplitude. As frequencies are added and shifted, it may happen that two very small terms can be driven into resonance. It is interesting to note that using the perturbative approach, the ultimate fate of the solar system is decided by the behaviour of very small terms on the order of $10^{-12}$.

\section{The KAM Theorem}

The properties of nonlinear oscillators near equilibrium points led Poincaré and others to investigate the topology of the phase space for Hamiltonian systems near equilibrium. The most fruitful approach was to classify equilibrium points, investigate their stability and discuss motion near these points. Birkhoff demonstrated the existence of both stable and unstable equilibrium points whenever there exists a rational frequency ratio between two frequences in a system. Work by Liapunov, Smale and others yielded nonlocal criteria for stability but these required the formulation of a function which depends on the details of the dynamical system, and for which a general recipe is not available. One must therefore rely on perturbation theory for the formulation. Kolmogorov, Arnold and Moser (KAM) used superconvergent methods to analyse the topology of the phase space for Hamiltonian systems perturbed away from integrable ones and the KAM theorem gives a qualitative description of the behaviour of phase space near equilibrium points. In understanding the theorem, it is useful to recall that in a Hamiltonian system, the state of the system is given by a state vector of $2 n$ dimensions, where $n$ is the number of degrees of freedom. The vector contains the generalized coordinates

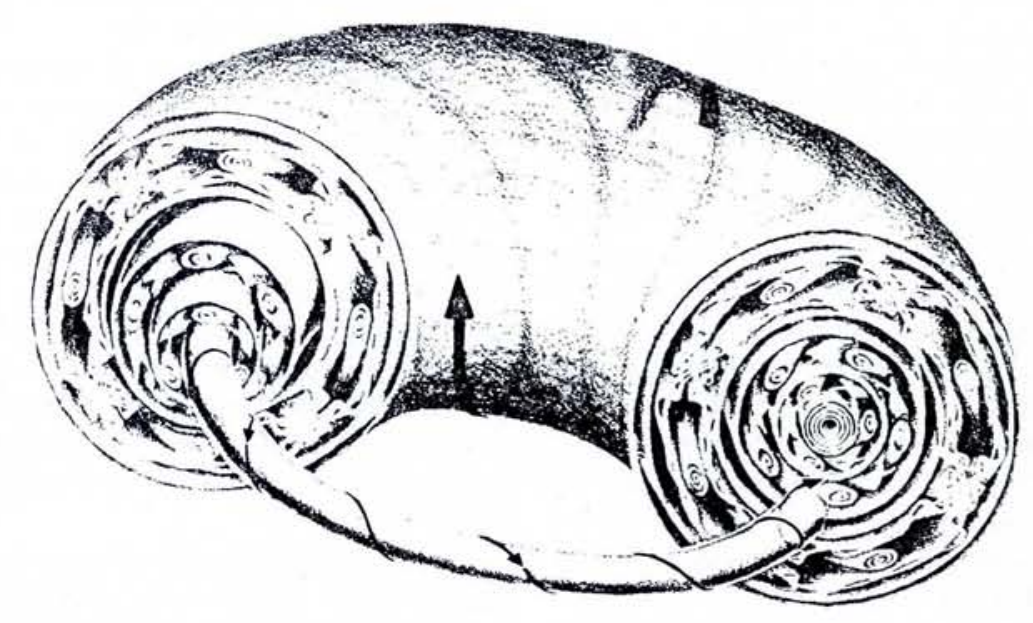

Fig. 1 - Illustration of KAM tori (from Ref. 3): as a non-linear system is more strongly perturbed away from equilibrium, the tori describing the state vector variables begin to intersect and to divide the phase space into empty and full cells. 

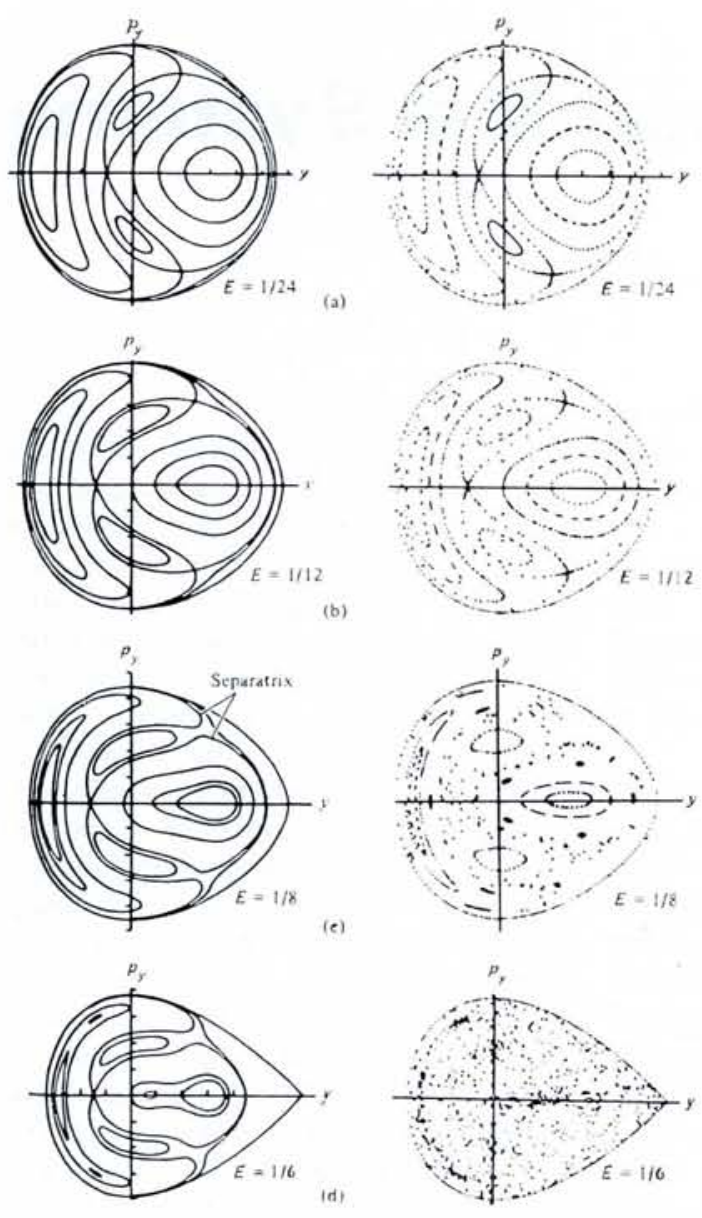

and momenta and its evolution with time, $t$, is given by

$d x / d t=f(x, t) ; x=\left(q_{1} \ldots q_{n}, p_{1} \ldots p_{n}\right)$

For an autonomous system, $f$ does not explicitly depend on time so the state of the system is in a $(2 n-1)$ dimensional subspace. It is possible to look at a Poincaré surface of section by examining an arbitrary projection of $x$ in a $2 n-2$ dimensional subspace. The evolution of the system can be traced as the set of points, $y$, on the surface of a section with each point $y_{i}$ derived from the previous one by an iteration,

$$
y_{i+1}=F\left(y_{i}\right)
$$

A fixed point is a point which goes into itself under the iteration. In an integrable system there will be additional relations between the state vector variables such that the orbits fall on tori in the phase space, or on closed curves on the surface of a section. A fixed point is the limiting point of recurring orbits, with an elliptic stable fixed point being surrounded by a family of nonintersecting periodic orbits and a separatrix; and a hyperbolic fixed point being stable in only one direction.

For example, in the case of a pendulum, the lower position is an elliptic fixed point; it is surrounded by integral curves of given energy. When the energy is sufficiently large, a second unstable equilibrium point is reached at

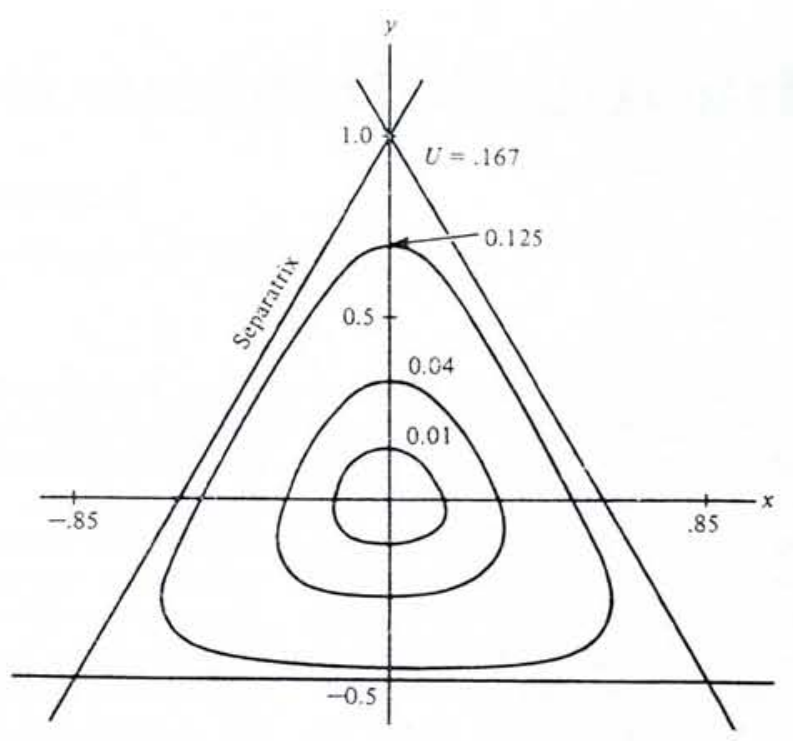

Fig. 2 - The surfaces of sections (left figure) for the Henon-Heiles potential (upper figure) plotted at various energy levels, E, showing that the phase space divides into empty and full cells as the energy increases. the upper position. This is a hyperbolic fixed point where the motion can either continue as an oscillation (if the pendulum turns back), or as a rotation (if it carries on in the same direction).

The KAM theorem states that along an elliptic fixed point, the majority of the tori maintain their topology if the system is perturbed away from equilibrium. However, the tori begin to intersect and divide the phase space into cells or chains as the perturbation grows stronger. Some of the cells are empty while some of them are full so points collect in them at random (Fig. 1).

Two possible sources of misunderstanding of the theorem must be clarified. First, it is not a "proof" of the ergodic theorem since a finite number of the trajectories remain regular. Furthermore, there exist non-Hamiltonian systems (where the theorem is not valid) in which ergodicity can be proved. Second, it cannot be the basis of a "hidden variable" approach to quantum mechanics, because the standard quantum prescriptions apply to integrable systems.

The KAM theorem did not attract the attention of the physics community until advances in numerical techniques enabled one to investigate (numerically) the behaviour of coupled nonlinear oscillators. It turned out that rather sim- ple analyses could be used to model meteorological, turbulent and astrophysical phenomena. However, their behaviour was anything but simple. As an example, Fig. 2 shows the surfaces of sections for the Henon-Heiles potential which is a two dimensional system with the Hamiltonian

$$
\begin{aligned}
H= & p_{x}^{2} / 2+p_{y}^{2} / 2+\left(x^{2}+y^{2}\right) / 2 \\
& +x^{2} y-y^{3} / 3=E
\end{aligned}
$$

The Figure gives bound orbits for $E \leq$ $1 / 6$ and it is clearly seen that there are definite orbits at low energies. As the energy increases, the phase space divides into empty and full cells.

\section{A Revolution in Chaos?}

These interesting results attracted considerable attention and scientists began to realize the extent to which apparently "simple" systems produced seemingly random or "chaotic" behaviour. Some authorities have claimed that this has led to a "chaos revolution" in that the way science views nature has changed. A popular book [1] by Gleick gives a detailed and exciting, but perhaps over-romanticised, exposition of these developments. He suggested that along with relativity and quantum mechanics, chaos is one of the three revolutions for which this century will be remembered. 
It is, perhaps, an overstatement to assert that chaos itself represents a scientific revolution. In fact an interdisciplinary area referred to by names such as nonlinear dynamics and chaos theory has grown rapidly owing to developments in computer technology and in the use of geometrical and topological methods $[2,3]$. These powerful tools enabled researchers to attack difficult problems in dynamics. Developments in computer hardware gave us both the supercomputers which could perform a very large number of calculations in a short time, and the personal calculators and computers which put inexpensive computing power in everyone's hand. Parallel developments in software yielded algorithms for the integration of systems over long intervals of time, the simulation of systems, and the graphical demonstration of results. Finally, new mathematical techniques gave the reassurance that computer results were correct.

The results are surprising. Nonintegrable problems with as few as two degrees of freedom have yielded phase spaces containing regions in which some properties of multiple orbits are random. On the other hand, simulations of systems comprising masses connected by nonlinear springs gave results

\section{Third Annual Complex Systems Summer School}

$$
\text { June 4-29, } 1990
$$

For graduate students and postdoctoral fellows, an intensive introduction to "complex" behavior in mathematical, physical and living systems. Topics include learning algorithms; stochastic processes in the physical and biological sciences, connectionist models of cognition; pattem formation in chemical systems; computational complexity. Subsistence stipends available. Application deadline: March 1, 1990.

\section{Co-Directors:}

Lynn Nadel, Psychology,

University of Arizona

Daniel Stein, Physics,

University of Arizona

Information:

Andi Sutherland

Santa Fe Institute, 1120 Canyon

Road, Santa Fe, NM 87501

505-984-8800; fax 505-982-0565

email:

summerschool@sfi.santafe.edu that are inconsistent with the equipartition hypothesis since, under certain initial conditions, the energy is concentrated within a few selected modes. This corresponds to a collective behaviour of the system which, in spite of the system's many degrees of freedom, is not "chaotic".

It is now understood that chaotic behaviour results from a strong dependence on initial conditions. If an error propagates exponentially as a dynamical system develops in time, then nearby trajectories diverge exponentially and the orbit depends sensitively on the initial state. A very small randomness (due to an error of measurement for instance) in the initial state is sufficient for this to occur.

It is also now realised that period doubling is one of the most prominent mechanisms for transitions from order to chaos. Feigenbaum has shown the universality of the period doubling mechanism and it has become clear that problems in several different fields of physics (e.g. response in semiconductor junctions, the behaviour of a hydrogen atom in a magnetic field, motion of electrons in axi-symmetric crystal fields, molecular vibrations, turbulence, atmospheric physics, plasma dynamics, evolution of the so-called "mixmaster universe" - an homogeneous, anisotropic, Bianchi-type cosmological solution to Einstein's equations) all exhibit this type of chaotic behaviour. In a way, the revolution is not chaos itself, but the various advances which have permitted scientists in different fields to formulate and solve nonlinear models.

\section{Chaotic Phenomena}

It is not easy to give a precise meaning to the term "chaos". Some of the different phenomena which can be labelled "chaotic" are [4]:

a) Irregular behaviour arising from the stochastic nature of a process, e.g. nuclear decay;

b) Irregular behaviour arising from sources of noise external to a system;

c) Irregular behaviour arising from the stochastic nature of the model used for a process. For example, in trying to understand the behaviour of a gas, it is practically impossible to write equations of motion for each of the $10^{20}$ atoms involved. Even if one develops supercomputers and algorithms suitable for the solution of such an enormous system, and we obtain the expressions for the time dependence of the coordinates of each atom, it is not clear how this information can be used meaningfully without taking a statistical average. If one is committed to a statistical interpretation, one may as well adopt a statistical approach at the outset. An hypothesis such as stosszahlenansatz (collision number ansatz) must be accepted, committing one to follow the macroscopic states of the system and leave the microscopic states in a state of atomic or molecular chaos.

d) Irregular behaviour arising from a natural or artificial limitation on the means for observing a system. Natural limitation is typified by the uncertainity principle and an artificially imposed limitation by the observation of an otherwise deterministic system in a coarse grained space. The most frequently quoted example of the latter is the doubling map given by $x_{n+1}=2 x_{n} \bmod 1$. The equation is completely deterministic, with the solution $x_{n}=2^{n} x_{0}$ mod 1 . A simple way to understand this solution is to expand $x_{0}$ in a binary fraction where each of the $x^{\prime}$ 's are obtained by right-shifting this binary fraction and discarding the integer part. Clearly, $\mathrm{O} \leq$ $x_{n}<1$. If one chooses to observe the system in a "coarse grained" space of two cells, $0 \leq x<1 / 2$ and $1 / 2 \leq x<$ 1 , the sequence of results is quite random in that it is generally impossible to predict a digit from the one before it for a non-rational starting number.

e) Irregular behaviour which does not

\section{Mathematica $^{\mathrm{TM}}$ \\ A System for Doing Mathematics by} Computer

\section{A Wolfram Research Inc. product}

$\square$ Numerics - Works with numbers of arbitrary magnitude and precision.

$\square$ Symbolics - Encyclopaedia of mathematical functions and operations used in arithmetic, algebra and analysis.

Procedural, functional and mathematical programming.

$\square$ Graphics - 2D, 3D and animated PostScript graphics.

$\square$ Text processing - Fully interactive reports and textbooks.

$\square$ Runs on - MS-DOS based computers; Macintosh, Apollo, Hewlett Packard, IBM AIX/RT, MIPS, Silicon Graphics, Sony, Sun, VAX.

Now available in Europe from:

MathSoft Overseas, Inc.

POB 641, 1211 Geneva 3, Switzerland Tel. ++41 (22) 465260

Fax $++41(22) 465939$ 
involve any of the causes mentioned above. In such systems, chaotic behaviour appears without requiring the presence of additional noise sources or stochastic processes.

\section{A Unifying Principle}

In physics, one usually looks for unifying principles, as for example, the Laplace equation that makes a linear theory applicable to many fields. It is remarkable that the nonlinear effect in all of the phenomena listed above can be described in terms of simple models that lead to chaotic behaviour. On a microscopic level, the origin of chaos lies in the sensitive dependence on initial conditions, and the assertion of an inability to make measurements that are more precise than those implied by the uncertainity principle.

What then is the attribute which initiates a sensitive dependence on initial conditions? We have seen that integrability makes the orbits of a dynamical system lie on tori in phase space. We also know that continuous symmetry in a problem leads to the existence of an integral; when symmetry is broken, the system loses the integral associated with the symmetry and chaotic behaviour sets in. The detailed mechanism of symmetry breaking may differ from one case to another, but the gross features can be described in terms of chaos. It is the breaking of rotational symmetry in 2, 3 or 4 dimensions that is one of the common characteristics in many chaotic problems cited above.

The motion of a hydrogen atom in a magnetic field [5] is a simple problem which illustrates all of these properties. Furthermore, it is not a contrived model but a truly physical system where it is possible to compare theoretical calculations with experimental observations. The transitions from order to chaos depend on a single parameter, namely the magnetic field strength, which can be experimentally adjusted. The Hamiltonian (where $y$ is related to the strength of the magnetic field)

$$
\begin{aligned}
H & =p^{2} / 2-1 / r+y L x / 2 \\
& +y^{2}\left(x^{2}+y^{2}\right) / 8=E
\end{aligned}
$$

shows the transition from a fully spherical symmetry to cylindrical symmetry as the magnetic field is turned on. Upon introducing semi-parabolic coordinates, one obtains the Hamiltonian

$H_{\mathrm{o}}=p_{u}^{2} / 2+p_{v}^{2} / 2+\left(u^{2}+v^{2}\right) / 2$

$+\lambda u^{2} v^{2}\left(u^{2}+v^{2}\right) / 8=2 \varepsilon \quad$ where $\lambda=y^{2} /(-2 E)^{1 / 2}$ and $\varepsilon=1 /(-2 E)^{1 / 2}$. This Hamiltonian has a scaling invariance so that the classical dynamics depend only on one parameter, $\beta=\lambda \varepsilon^{2}=$ $y^{2} /(-2 E)^{3 / 2}$. Poincaré surfaces of sec-
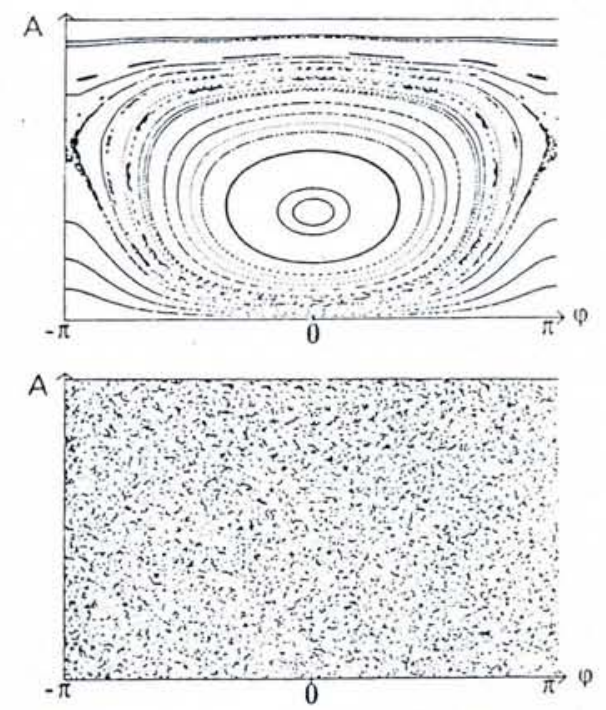

Fig. 3 - Poincaré surfaces of sections for a hydrogen atom in a magnetic field. Surfaces with $p_{y}=0$ are plotted using $E_{u}$ and $\phi$ is coordinates. Motion is nearly regular for $\beta=0.8$ (upper) and becomes fully chaotic on increasing $\beta$ to 70 (from Ref. 5).

tions for $p_{y}=0$ using $E_{u}=p^{2}+u^{2}$ versus $\phi=\arctan \left(p_{\mathrm{u}} / u\right)$ as coordinates are plotted in Fig. 3 for $\beta=0.8$ and $\beta=$ 70 (taken from Ref. 5). The transition from a nearly regular motion with a few KAM regions to fully chaotic motion is apparent. The fixed point corresponds to a circular orbit in a plane perpendicular to the magnetic field.

It should be mentioned that chaotic behaviour in Hamiltonian systems results from a transition from an integrable to a non-integrable system under perturbations which break the symmetry that leads to the integral. An integral places the trajectory on a subspace of the phase space. In non-Hamiltonian systems, the Liouville theorem is not applicable and dissipation will cause one to access a subspace of the phase space. Forced nonlinear systems with dissipation will access trajectories called strange attractors in such subspaces. Sets of nearby trajectories end up in these attractors so information about initial conditions is lost.

\section{Conclusions}

The stability properties of systems must be well understood in order to study the transition to chaos. Another important aspect is the extraction of information about a system from experimentally observed frequency data. In spite of these shortcomings, recent developments in the theory of chaos permit a better understanding of nonlinear systems. It is necessary to let some of them filter into physics curricula where undergraduate instruction in classical mechanics presently concentrates on linear, integrable systems.
Acknowledgement

The author acknowledges useful discussions with Professor Y. Nutku.

\section{REFERENCES}

[1] Gleick J., Chaos, Making a New Science (Penguin Books, New York) 1988.

[2] Lichtenberg A.J. and Lieberman M.A., Regular and Stochastic Motion (Springer Verlag, New York) 1983.

[3] Arnold V.J., Mathematical Methods of Classical Mechanics (Springer Verlag, New York) 1978; Abraham R. and Marsden J.E., Foundations of Mechanics (The Benjamin/ Cummings Publishing Co., Inc., Reading, Mass.) 1978.

[4] Cvitanovic P., Universality in Chaos (Adam Hilger Ltd, Bristol) 1984.

[5] Delande D. and Gray J.C., Phys. Rev. Lett. 57 (1986) 2006; Harada A. and Hasegawa H., J. Phys. 16A (1983) L259.

\section{Chaotic Software}

The first Physics CTI (Computers in Teaching Initiative) Newsletter and Version 1 of the Physics Courseware Catalogue were issued last September. They can be obtained by contacting the Physics CTI Centre, Department of Physics, University of Surrey, Guildford, GU2 $5 \mathrm{XH}$, UK (Tel. ++44 483 509329). The catalogue gives the availability (in the UK only) and a short description of each of nearly 70 different computer software packages for teaching physics that have been announced to the Centre. They are classified into 11 categories such as optics, quantum mechanics, etc.

Readers of our article on chaotic behaviour in dynamical systems by Professor Hacinliyan may be interested to note that the catalogue describes six packages for modelling examples of these systems, especially the simple pendulum. For instance, various effects discussed in the article such as period doubling leading to chaos for a damped, driven pendulum can be studied using a programme developed by Salford University.

The catalogue concentrates on software developed at British universities for BBC personal computers and for IBM compatibles. Future versions will describe the more commercial software, as well as the increasing number of programmes available in the US for the Mackintosh. The Centre would also like to include details of European software for teaching physics that is available in languages other than English. Information should be sent directly to the Physics CTI Centre.

\section{Research Facilities}

The combined proceedings of the four EPS Seminars on International Research Facilities provide a definitive survey of the big physics facilities in the building or planning phases. The set comprising the Zagreb (1989), London (1986), Copenhagen (1983) and Rome (1979) proceedings are available from the EPS Secretariat for 120 SFR $(60$ SFR to IOM's). Individually, they cost 60 , 65,25 and 60 SFR respectively (half price to IOM's). 